\title{
Computação Quântica Adiabática: Do Teorema Adiabático ao Computador da D-Wave
}

\author{
Adiabatic Quantum Computation: From Adiabatic Theorem to D-Wave Computer
}

\author{
Paulo J. P. Souza ${ }^{*}$, Taysa M. Mendonça ${ }^{1}$, Estêvão V. B. de Oliveira ${ }^{1}$, Celso J. Villas-Boas ${ }^{1}$ \\ ${ }^{1}$ Universidade Federal de São Carlos, Departamento de Física, São Carlos, SP, Brasil.
}

\begin{abstract}
Recebido em 01 de Fevereiro de 2021. Revisado em 16 de Fevereiro de 2021. Aceito em 17 de Fevereiro de 2021.
Neste artigo descrevemos os fundamentos da computação quântica adiabática, que consiste na interpolação de diferentes Hamiltonianos, desde um Hamiltoniano inicial, com autoestados e autoenergias conhecidos, até um Hamiltoniano final, onde é codificada a solução de um dado problema. Em particular, discutimos o método do annealing quântico, que é utilizado pela empresa canadense D-Wave, pioneira nessa área. Primeiramente apresentamos uma das demonstrações do teorema adiabático quântico e de uma condição de adiabaticidade para evolução de sistemas quânticos. Para exemplificar, descrevemos a implementação do algoritmo de Grover via computação quântica adiabática, discutindo os impactos da escolha da forma de interpolação dos Hamiltonianos inicial e final na complexidade do algoritmo. Em seguida, mostramos um algoritmo para o problema do caixeiro viajante executável na plataforma da D-Wave. Por fim, apresentamos aspectos físicos importantes da tecnologia dos computadores quânticos da D-Wave, expondo suas vantagens e desvantagens, além de descrever qualitativamente a engenharia de seu processador quântico.
\end{abstract}

Palavras-chave: Computação quântica adiabática, Annealing quântico, empresa D-Wave.

In this paper we describe the fundamentals of the adiabatic quantum computing, which consists of the interpolation of different Hamiltonians, from an initial Hamiltonian, with known eigenstates and eigenenergies, to a final Hamiltonian, in which the solution to a given problem is encoded. In particular, we discuss the quantum annealing method, which is used by the Canadian company D-Wave, a pioneer in this area. Firstly, we present the proof of the quantum adiabatic theorem and an adiabaticity condition for the evolution of quantum systems. To exemplify, we describe the implementation of the Grover algorithm via adiabatic quantum computing, discussing the impacts of the choice of the interpolation form of the initial and final Hamiltonians on the complexity of the algorithm. In the following, we show an algorithm for the travelling salesman problem executable on the D-Wave platform. Finally, we present important physical aspects of D-Wave's quantum computer technology, exposing its advantages and disadvantages, in addition to qualitatively describing the engineering of its quantum processor.

Keywords: Adiabatic quantum computing, Quantum Annealing, D-Wave company.

\section{Introdução}

A teoria da computação foi concebida em 1935 por Alan Turing [1] e foi essencial para que os Países Aliados tivessem êxito na segunda guerra mundial. Em 1945, o primeiro computador de larga escala foi construído, o Electronic Numerical Integrator and Compute (ENIAC), que processava 5 mil informações por segundo e era composto por válvulas térmicas que ocupavam uma sala de $167 \mathrm{~m}^{2}$ [2] Na década de 1950, a primeira linguagem de computação imperativa foi desenvolvida, o FORTRAN. Essa linguagem computacional é ainda muito utilizada, principalmente em computação numérica [3]. O primeiro computador pessoal surgiu em 1981 e foi lançado pela International Business Machines Corporation ou, simplesmente, IBM. O IBM 5150 possuía $22 \mathrm{~kg}$ e conseguia realizar 4.7 milhões de operações por segundo, contava com uma memória RAM de $16 \mathrm{kB}$ e

\footnotetext{
*Endereço de correspondência: paulo.paulino.souza96@gmail.com
}

nenhum disco rígido 4. Hoje, quase 40 anos depois, temos computadores pessoais com capacidade de processamento milhões de vezes maior e com capacidades de armazenamento de enormes quantidades de dados.

A história da Computação Quântica começou na década de 1980 quando Paul Benioff [5] e Richard Feynman [6] mostraram que sistemas quânticos poderiam oferecer algo novo para a computação. Segundo Feynman, nenhuma máquina de Turing (clássica) seria capaz de simular alguns fenômenos quânticos sem introduzir um fator que cresce exponencialmente com o tamanho do sistema em seu desempenho. Essas contribuições serviram para conceber a ideia das áreas de pesquisa conhecidas hoje como Computação e Simulação Quânticas. Em 1999 foram construídos os primeiros protótipos de computadores quânticos [7] mas apenas em 2011 a D-Wave lançou o primeiro computador quântico comercial 8 .

A computação clássica tem como unidade fundamental de informação o bit, que pode possuir valores ' 1 ' 
ou ' 0 ', que podem ser referentes a ter ou não ter corrente elétrica passando por um determinado circuito. Em analogia ao bit, na computação quântica tem-se como unidade básica o q-bit - ou qubit, do inglês quantum bit. Um q-bit pode ser qualquer sistema quântico com dois estados de energia bem definidos, sendo $|0\rangle$ e |1) associados aos estados de menor e maior energia, respectivamente. $\mathrm{O}$ poder da computação quântica vem do fato de que, diferentemente do bit clássico, o bit quântico pode estar em uma superposição de estados, ou seja, pode assumir os valores zero e um ao mesmo tempo 9 .

Experimentos de computação quântica podem ser realizados usando diferentes técnicas [10], como por exemplo os circuitos supercondutores [11, 12, íons aprisionados [13], pontos quânticos [14], etc. Tais experimentos são realizados em dois tipos distintos de programação: por portas lógicas [15] e computação adiabática 16]. A computação quântica por portas é muito similar às portas lógicas clássicas, já a computação quântica adiabática (CQA) usa de interpolações adiabáticas. Como a computação quântica é reversível, as duas formas de computação são equivalentes. De fato, a própria e D-Wave anunciou recentemente uma ferramenta que fornece interoperabilidade entre o computadores quânticos que funcionam usando a técnica de annealing e os computadores quânticos do modelo de porta [17].

Os computadores quânticos ainda estão na era NISQ (Noisy Intermediate-Scale Quantum) [18], o que significa que ainda estamos com o controle parcial das dificuldades impostas pelos ruídos e decoerências em sua operação. Isso implica que ainda não é possível mostrar na prática a superioridade do desempenho dos computadores quânticos em problemas reais. Essas dificuldades de engenharia estão sendo superadas pouco a pouco, o que tem levado ao constante aumento no número de q-bits em operação com o passar das gerações de computadores. Com isso, hoje presenciamos uma grande corrida internacional visando a construção de computadores quânticos, motivando governos e empresas multinacionais a investirem bilhões de dólares nessa área, culminando com os anúncios recentes de uma equipe da Google 19 e de uma equipe liderada pelo Prof. Jian-Wei Pan [20], que alegam terem enfim obtido a tão sonhada supremacia quântica 1 Por outro lado, mais recentemente a D-Wave anunciou seu novo computador quântico Advantage 22 que, segundo a empresa, permite resolver alguns problemas práticos com similar desempenho em relação aos dispositivos clássicos.

\footnotetext{
1 Supremacia quântica é um termo introduzido por John Preskill 21] para designar máquinas quânticas capazes de executar tarefas práticas que computadores clássicos não são capazes. Embora os dois grupos tenham obtidos resultados que, segundo eles, não sejam factíveis atualmente mesmo nos maiores supercomputadores clássicos [19, 20], as tarefas executadas por essas equipes ainda não tem interesse prático e, portanto, não teriam ainda atingido a supremacia quântica, como definida por J. Preskill.
}

Nesse trabalho temos como objetivo a divulgação e apresentação da teoria da computação quântica adiabática e algumas aplicações. Começaremos apresentando como se dá a resolução de problema via interpolações adiabáticas, depois anunciamos o teorema adiabático e derivamos a condição de adiabaticidade tradicional da literatura [23 25]. Como exemplos, apresentamos dois algoritmos de computação adiabática: um que reproduz o algoritmo de busca de Grover [26, 27] e outro que resolve o problema do caixeiro viajante. Em seguida expomos a tecnologia que compõe os computadores quânticos que usam a técnica de annealing em sua programação. Em especial mostraremos o funcionamento dos computadores da empresa canadense D-wave, uma das mais bem sucedidas na área de computação quântica. Por fim, mostraremos qualitativamente a parte experimental do seu Hardware, como implementar alguns problemas na plataforma da empresa, o porquê de seu sucesso comercial e suas limitações.

\section{Computação Quântica Adiabática e Annealing Quântico}

Annealing, que em português pode ser traduzido como recozimento, tem significados similares nos mundos clássico e quântico. Problemas que envolvem annealing clássico, em geral, são problemas que envolvem termodinâmica, onde é definido um Hamiltoniano clássico para o sistema e as leis de transição entre os microestados do sistema 28]. Esse Hamiltoniano conecta as soluções do problema de otimização em forma de energias, sendo que a solução de menor energia é a solução ótima. Com isso, o sistema termodinâmico é iniciado em um estado aleatório e então a temperatura é reduzida adiabaticamente (de forma suficientemente lenta para que sempre seja garantido que o estado de maior entropia seja atingido), até valores próximos de $T=0 \mathrm{~K}$. Durante esse processo o sistema evolui gradualmente até atingir seu estado fundamental 29 .

Já o annealing quântico tem o propósito de encontrar um Hamiltoniano de menor energia, que é a solução do problema proposto, partindo de um Hamiltoniano inicial que possui seu espectro conhecido. A interpolação entre esses dois operadores deve ser realizada adiabaticamente. Nesse caso a adiabaticidade nada tem a ver com parâmetros termodinâmicos de um processo relacionado a trocas de calor mas sim com uma dinâmica de evolução quase estática onde não há transições entre os níveis de energia da evolução Hamiltoniana, como será mostrado no decorrer deste artigo.

Para um melhor entendimento da nomenclatura, devemos fazer a distinção entre computação quântica adiabática e annealing quântico, pois a computação é a formalização da teoria de algoritmos, assim como há o modelo da máquina de Turing. Já quando nos referimos ao annealing quântico estamos nos referindo ao processo físico que faz a transição adiabática. Em complemento, 
o nome annealing aparece, assim como em seu análogo clássico, pois os erros experimentais e pertubações do ambiente fazem com que a evolução do sistema físico não seja de forma idealmente adiabática, assim dando uma característica heurística ao algoritmo.

A computação adiabática é Turing completa, ou seja, usando-a é possível implementar qualquer algoritmo [30]. Entretanto, o aparato experimental da D-Wave, nos restringe a problemas que podem ser escritos como um problema tipo Ising ou QUBO ${ }^{2}$ como o problema do caixeiro viajante, solução de equações lineares, problemas de decisão, de empacotamento, entre outros.

Em termos gerais, algoritmos de Computação Quântica Adiabática (CQA), são descritos por um Hamiltoniano dependente do tempo parametrizado $s$

$$
\hat{H}(s)=A(s) \hat{H}_{0}+B(s) \hat{H}_{f},
$$

onde $\hat{H}_{0}$ e $\hat{H}_{f}$ são, respectivamente, os Hamiltonianos inicial e final, com $s \in[0,1]$, e $A(s)$ e $B(s)$ são as funções que descrevem a interpolação entre os Hamiltonianos e que obedecem às condições de contorno genéricas dadas por

$$
A(0) \neq 0, \quad B(1) \neq 0, \quad A(1)=B(0)=0 .
$$

Em geral, nos experimentos nem sempre é possível ajustar $A(1)$ e $B(0)$ identicamente iguais a zero. No entanto, é suficiente ajustar $A(0) \gg B(0)$ e $B(1) \gg A(1)$ para que o sistema parta de um estado conhecido e chegue ao estado fundamental do Hamiltoniano final (ao menos aproximadamente), desde que o processo de annealing seja feito de forma suficientemente lenta.

A complexidade computacional dos algoritmos de CQA depende do gap de energia entre o estado fundamental e o primeiro estado excitado, que em geral escalam com o número de q-bits envolvidos. Assim, para encontrar o gap é necessário encontrar o estado fundamental e primeiro estado exitado ao longo da evolução, o que é por si só um tarefa computacionalmente custosa. Desse modo, essa discussão fica limitada aos casos onde é possível obter o espectro do Hamiltoniano dependente do tempo analiticamente.

No caso específico da empresa D-Wave, o processador quântico possui um conjunto de q-bits acoplados em uma configuração equivalente a uma cadeia de spins magnéticos do tipo Ising. Nesse caso, o Hamiltoniano inicial do sistema é obtido aplicando-se campos magnéticos transversos de forma que os spins (q-bits) do sistema se orientem na direção do campo. A interpolação adiabática (processo $\hat{H}_{0} \rightarrow \hat{H}_{f}$ ) é feita reduzindo-se lentamente a intensidade do campo transverso a zero ao passo que a intensidade dos acoplamentos entre os q-bits e da anisotropia é aumentada. Com isso temos o

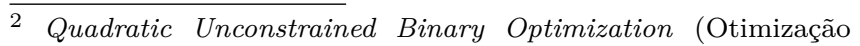
Binária Quadrática Irrestrita, em tradução livre).
}

Hamiltoniano dependente do tempo com a forma:

$\hat{H}(s)=A(s) \sum_{i} \hat{\sigma}_{i}^{x}+B(s)\left(\sum_{i, j} J_{i j} \hat{\sigma}_{i}^{z} \hat{\sigma}_{j}^{z}+\sum_{i} h_{i} \hat{\sigma}_{i}^{z}\right)$,

onde os operadores $\hat{\sigma}_{i}^{\alpha}$ correspondem às matrizes de Pauli $\alpha=x, y, z$ para o $i$-ésimo sítio, $J_{i j}$ é o acoplamento entre os sítios $i$ e $j$ e $h_{i}$ é o campo anisotrópico aplicado ao sítio $i$. O annealing linear define o fator $s=t / \tau_{\max }$, onde $t$ é o tempo e $\tau_{\max }$ é o tempo total de annealing. Nesse Hamiltoniano, a função $A(s)$ é conhecida como energia de tunelamento e a função $B(s)$ está associada ao "Hamiltoniano problema". Além disso, experimentalmente, $B(s=0) \neq 0$ de modo que, para obter as condições necessárias para se ter a evolução desejada, faz-se $A(0) \gg B(0)$.

A Figura 1 mostra a evolução das funções temporais $A(s)$ e $B(s)$ como função de $s$ para o sistema da D-Wave enquanto a temperatura física permanece constante [31]. $\mathrm{Na}$ prática, em $t=0, A(0)$ será máximo e $B(0)$ será aproximadamente zero, o que leva ao estado fundamental do sistema, que consiste no produto tensorial de cada spin em seu estado fundamental. À medida que o sistema evolui, $A(s)$ diminui e $B(s)$ aumenta até que, no final, o Hamiltoniano total contém o único termo, aquele associado à $B(s)$. Se o processo for lento o suficiente, ou seja, adiabático, o estado final dos q-bits corresponderá ao estado fundamental do Hamiltoniano final do sistema, o qual codifica uma solução para o problema em questão. Um ponto importante é: Para um dado sistema, qual condição garante a sua permanência em seu estado fundamental durante toda evolução? A resposta para essa pergunta nos é fornecida pelo teorema adiabático.

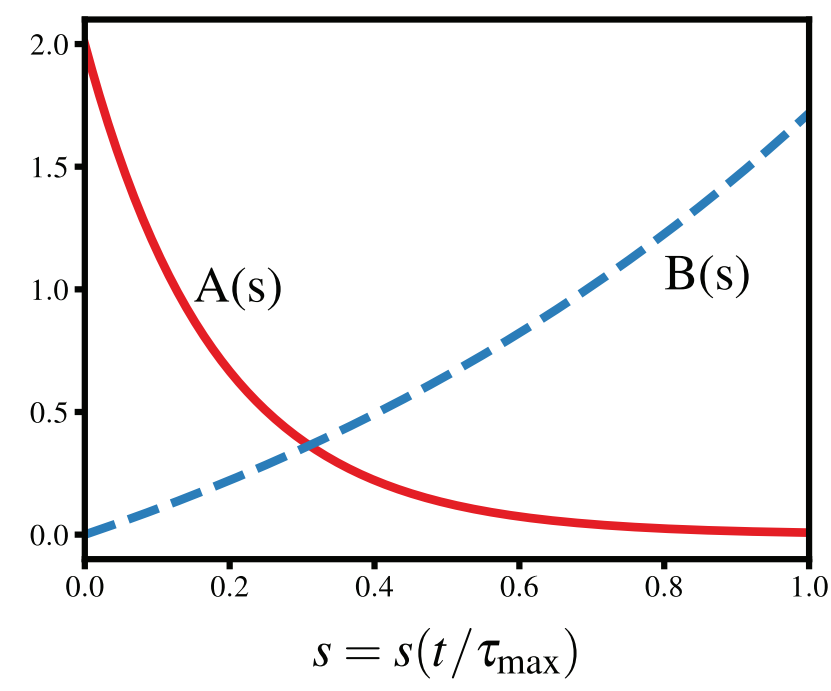

Figura 1: Evolução das funções $A(s)$ e $B(s)$. O annealing começa em $s=0 \mathrm{com} A(s) \gg B(s)$ e termina em $s=1 \mathrm{com}$ $A(s) \ll B(s)$. As funções escolhidas são arbitrárias, similares às utilizadas no $D$-Wave $2 X$ systems [31], respeitando apenas as condições de contorno descritas pela equação (2). 


\section{Teorema Adiabático}

O teorema adiabático, em sua versão mais conhecida, foi primeiramente demonstrado por Max Born e Vladimir Fock em 1928 [32]. Posteriormente, em 1950, uma demonstração mais rigorosa foi dada por Tosio Kato [33]. O enunciado de Born, conhecido como Teorema Adiabático, nos diz que

Teorema 1. Um sistema físico permanece em seu autoestado instantâneo, não degenerado, ao longo da evolução temporal, se sobre ele é aplicada uma pertubação suficientemente pequena.

Para provar o teorema adiabático, e posteriormente derivar um condição de adiabaticidade, consideramos a equação de autovalor com o operador Hamiltoniano dependente do tempo

$$
\hat{H}(t)\left|\psi_{n}(t)\right\rangle=E_{n}(t)\left|\psi_{n}(t)\right\rangle,
$$

onde $\left|\psi_{n}(t)\right\rangle$ e $E_{n}(t)$ são, respectivamente, os autoestados e autoenergias instantâneos do operador Hamiltoniano $\hat{H}(t)$. Podemos encontrar soluções mais gerais escrevendo a equação de Schrödinger dependente do tempo

$$
\hat{H}(t)|\Psi(t)\rangle=i \partial_{t}|\Psi(t)\rangle
$$

com $\hbar=1$, por simplicidade. Para o desenvolvimento a seguir, é conveniente escrever $|\Psi(t)\rangle$ como uma superposição dos autoestados do Hamiltoniano na forma

$$
|\Psi(t)\rangle=\sum_{n} c_{n}(t) e^{i \theta_{n}(t)}\left|\psi_{n}(t)\right\rangle,
$$

onde $\theta_{n}(t)$ é a denominada fase dinâmica e é dada por

$$
\theta_{n}(t)=-\int_{0}^{t} E_{n}\left(t^{\prime}\right) \mathrm{d} t^{\prime}
$$

Para uma maior clareza na notação, daqui em diante omitiremos a dependência temporal de todos os termos. Substituindo a equação (6) na equação (5) obtemos

$$
\hat{H} \sum_{n} c_{n} e^{i \theta_{n}}\left|\psi_{n}\right\rangle=i \partial_{t}\left(\sum_{n} c_{n} e^{i \theta_{n}}\left|\psi_{n}\right\rangle\right) .
$$

A derivada do termo à direita resulta em

$$
i \sum_{n}\left(\dot{c}_{n} e^{i \theta_{n}}\left|\psi_{n}\right\rangle+i \dot{\theta}_{n} c_{n} e^{i \theta_{n}}\left|\psi_{n}\right\rangle+c_{n} e^{i \theta_{n}}\left|\dot{\psi}_{n}\right\rangle\right) .
$$

Aplicando o operador Hamiltoniano obteremos então a igualdade

$$
\begin{aligned}
& \sum_{n} c_{n} e^{i \theta_{n}} E_{n}\left|\psi_{n}\right\rangle \\
& \quad=i \sum_{n}\left(\dot{c}_{n} e^{i \theta_{n}}\left|\psi_{n}\right\rangle-i E_{n} c_{n} e^{i \theta_{n}}\left|\psi_{n}\right\rangle+c_{n} e^{i \theta_{n}}\left|\dot{\psi}_{n}\right\rangle\right)
\end{aligned}
$$

que se reduz à equação

$$
\sum_{n} \dot{c}_{n} e^{i \theta_{n}}\left|\psi_{n}\right\rangle=-\sum_{n} c_{n} e^{i \theta_{n}}\left|\dot{\psi}_{n}\right\rangle
$$

Projetando essa equação em um dado autoestado arbitrário $\left|\psi_{m}(t)\right\rangle$ e explorando a ortonormalidade dos vetores da base, temos a equação diferencial que rege a dinâmica dos coeficientes $c_{m}(t)$ de um dado estado instantâneo

$$
\dot{c}_{m}=-c_{m}\left\langle\psi_{m} \mid \dot{\psi}_{m}\right\rangle-\sum_{n \neq m} c_{n} e^{i\left(\theta_{n m}\right)}\left\langle\psi_{m} \mid \dot{\psi}_{n}\right\rangle,
$$

$\operatorname{com} \theta_{n m}=\left(\theta_{n}-\theta_{m}\right)$.

Para obtermos o termo $\left\langle\psi_{m} \mid \dot{\psi}_{n}\right\rangle$ podemos tomar a derivada temporal da equação (4)

$$
\dot{\hat{H}}\left|\psi_{n}\right\rangle+\hat{H}\left|\dot{\psi}_{n}\right\rangle=\dot{E}_{n}\left|\psi_{n}\right\rangle+E_{n}\left|\dot{\psi}_{n}\right\rangle .
$$

Novamente, tomando o produto interno com $\left\langle\psi_{m}\right|$ na equação 13

$\left\langle\psi_{m}|\dot{\hat{H}}| \psi_{n}\right\rangle+E_{m}\left\langle\psi_{m} \mid \dot{\psi}_{n}\right\rangle=\dot{E}_{n}\left\langle\psi_{m} \mid \psi_{n}\right\rangle+E_{n}\left\langle\psi_{m} \mid \dot{\psi}_{n}\right\rangle$,

e para o caso em que $E_{n} \neq E_{m}$, temos

$$
\left\langle\psi_{m} \mid \dot{\psi}_{n}\right\rangle=\frac{\left\langle\psi_{m}|\dot{\hat{H}}| \psi_{n}\right\rangle}{\left(E_{n}-E_{m}\right)} .
$$

Substituindo o resultado acima na equação 12

$$
\dot{c}_{m}=-c_{m}\left\langle\psi_{m} \mid \dot{\psi}_{m}\right\rangle-\sum_{n \neq m} c_{n} e^{i \theta_{n m}} \frac{\left\langle\psi_{m}|\dot{\hat{H}}| \psi_{n}\right\rangle}{\left(E_{n}-E_{m}\right)} .
$$

A equação 16 é exata e sua dinâmica tem contribuição de dois termos: o primeiro diz respeito à evolução de $c_{m}$ e leva em conta a variação do $m$-ésimo autoestado no tempo, enquanto o segundo representa um termo de mistura entre o $m$-ésimo e $n$-ésimo autoestados que dependem da evolução temporal do operador Hamiltoniano e de suas diferentes energias.

A aproximação adiabática consiste em impor certas condições ao sistema de modo a tornar o segundo termo, o termo de mistura entre os autovetores, negligenciável. Isso pode ser obtido se impusermos que a taxa de variação de $\hat{H}(t)$ é ínfima com relação à diferença de energia $E_{n m} \equiv E_{n}-E_{m}$ entre o $m$-ésimo e o $n$-ésimo estado. Nessa condição, a equação (16) pode, então, ser reescrita como

$$
\dot{c}_{m}=-c_{m}\left\langle\psi_{m} \mid \dot{\psi}_{m}\right\rangle,
$$

assim, resolvendo a equação acima,

$$
c_{m}(t)=c_{m}(0) e^{i \gamma_{m}},
$$

onde

$$
\gamma_{m}=i \int^{t}\left\langle\psi_{m} \mid \dot{\psi_{m}}\right\rangle \mathrm{d} t^{\prime}
$$

é denominada fase geométrica do sistema. 
Substituindo a equação (18) na equação (6), o vetor de estado $|\Psi\rangle$ em uma dinâmica adiabática será dado por

$$
|\Psi(t)\rangle=\sum_{m} c_{m}(0) e^{i \theta_{m}} e^{i \gamma_{m}}\left|\psi_{m}(t)\right\rangle
$$

onde vemos que os coeficientes são desacoplados e não há mistura de estados durante a evolução temporal. Portanto, temos que se as condições de adiabaticidade forem obedecidas, a equação 20 mostra que o sistema, partindo de um dado autoestado, permanece em seu autoestado instantâneo durante toda a evolução temporal.

\subsection{Condição de Adiabaticidade}

Nessa seção vamos analisar mais detalhadamente a condição adiabática, onde levamos em conta que a equação (18) é um resultado aproximado da equação (16). Para resolver a equação (16), introduziremos um fator integrante $\mu(t)$. Rearranjando os termos, teremos

$$
\begin{aligned}
& \mu(t) \dot{c}_{m}+\mu(t) c_{m}\left\langle\psi_{m} \mid \dot{\psi}_{m}\right\rangle \\
& =-\mu(t) \sum_{n \neq m} c_{n} e^{i \theta_{n m}} \frac{\left\langle\psi_{m}|\dot{\hat{H}}| \psi_{n}\right\rangle}{E_{n m}},
\end{aligned}
$$

que toma a forma

$$
\mu(t) \dot{c}_{m}-i \mu(t) c_{m} \dot{\gamma}_{m}=-\mu(t) \sum_{n \neq m} c_{n} e^{i \theta_{n m}} \frac{\left\langle\psi_{m}|\dot{\hat{H}}| \psi_{n}\right\rangle}{E_{n m}}
$$

Assumindo então que

$$
\mu(t) \dot{c}_{m}-i \mu(t) c_{m} \dot{\gamma}_{m}=\frac{\mathrm{d}}{\mathrm{d} t}\left[\mu(t) c_{m}\right],
$$

aplicando a regra da cadeia e resolvendo a equação (23) obtemos que $\mu(t)=e^{-i \gamma_{n}}$. Substituindo na equação (21), obtemos

$$
\frac{\mathrm{d}}{\mathrm{d} t}\left(c_{m} e^{-i \gamma_{m}}\right)=-\sum_{n \neq m} c_{n} e^{i\left(\theta_{n m}-\gamma_{m}\right)} \frac{\left\langle\psi_{m}|\dot{\hat{H}}| \psi_{n}\right\rangle}{E_{n m}} .
$$

Nesta etapa é interessante reparametrizar o tempo a fim de introduzirmos a escala de tempo referente à passagem adiabática. Fazendo

$$
s=\frac{t}{\tau_{\max }},
$$

com $\tau_{\max }$ sendo o tempo total da evolução temporal. Reparametrizando por $s$ a equação (24) temos

$$
\partial_{s}\left(c_{m} e^{-i \gamma_{m}}\right)=-\sum_{n \neq m} c_{n} e^{i\left(\tau_{\max } \omega_{n m}-\gamma_{m}\right)} \frac{\left\langle\psi_{m}\left|\partial_{s} \hat{H}\right| \psi_{n}\right\rangle}{E_{n m}},
$$

com

$$
\gamma_{m}(s)=i \int^{s}\left\langle\psi_{m}\left|\partial_{s}\right| \psi_{m}\right\rangle \mathrm{d} s^{\prime}
$$

e

$$
\omega_{n m}(s)=-1 \int^{s} E_{n m}\left(s^{\prime}\right) \mathrm{d} s^{\prime} .
$$

Definindo a variável

$$
F_{m n}(s)=c_{n} e^{-i \gamma_{m}}\left\langle\psi_{m}\left|\partial_{s} \hat{H}\right| \psi_{n}\right\rangle
$$

e integrando a equação 26 em relação ao tempo parametrizado, obtemos

$$
\begin{aligned}
& \int_{0}^{s} \partial_{s}\left[c_{m}\left(s^{\prime}\right) e^{-i \gamma_{m}\left(s^{\prime}\right)}\right] \mathrm{d} s^{\prime} \\
& \quad=-\sum_{n \neq m} \int_{0}^{s} \frac{F_{n} m\left(s^{\prime}\right)}{E_{n m}\left(s^{\prime}\right)} e^{i \tau_{\max } \omega_{n m}\left(s^{\prime}\right)} \mathrm{d} s^{\prime} .
\end{aligned}
$$

Notemos que o integrando do lado direito da equação 30 pode ser reescrito como

$$
\begin{aligned}
& \frac{F_{m n}\left(s^{\prime}\right)}{E_{m n}\left(s^{\prime}\right)} e^{i \tau_{\max } \omega_{m n}\left(s^{\prime}\right)} \\
& \quad=\frac{F_{m n}\left(s^{\prime}\right)}{\left[E_{m n}\left(s^{\prime}\right)\right]^{2}} E_{m n} e^{i \tau_{\max } \omega_{m n}\left(s^{\prime}\right)} \\
& =\frac{-i}{\tau_{\max }} \frac{F_{m n}\left(s^{\prime}\right)}{\left[E_{m n}\left(s^{\prime}\right)\right]^{2}} \partial_{s^{\prime}}\left[e^{i \tau_{\max } \omega_{m n}\left(s^{\prime}\right)}\right] .
\end{aligned}
$$

Por fim, substituindo o resultado da equação (31) na equação (30) e aplicando a técnica de integração por partes, temos

$$
\begin{aligned}
& {\left[c_{m}\left(s^{\prime}\right) e^{-i \gamma_{m}\left(s^{\prime}\right)}\right]_{0}^{s}} \\
& =\frac{i}{\tau_{\max }} \sum_{n \neq m}\left\{\left[\frac{F_{m n}\left(s^{\prime}\right)}{\left[E_{m n}\left(s^{\prime}\right)\right]^{2}} e^{i \tau_{\max } \omega_{m n}\left(s^{\prime}\right)}\right]_{0}^{s}\right. \\
& \left.\quad-\int_{0}^{s} e^{i \tau_{\max } \omega_{m n}\left(s^{\prime}\right)} \partial_{s^{\prime}}\left[\frac{F_{m n}\left(s^{\prime}\right)}{\left[E_{m n}\left(s^{\prime}\right)\right]^{2}}\right] \mathrm{d} s^{\prime}\right\},
\end{aligned}
$$

e ainda,

$$
\begin{aligned}
& c_{m}(s) e^{-i \gamma_{m}(s)}-c_{m}(0) \\
& =\frac{i}{\tau_{\max }} \sum_{n \neq m}\left\{\left[\frac{F_{m n}(s)}{\left[E_{m n}(s)\right]^{2}} e^{-i \tau_{\max } \omega_{m n}(s)}-\frac{F_{m n}(0)}{\left[E_{m n}(0)\right]^{2}}\right]\right. \\
& \left.\quad+-\int_{0}^{s} e^{i \tau_{\max } \omega_{m n}\left(s^{\prime}\right)} \partial_{s^{\prime}}\left[\frac{F_{m n}\left(s^{\prime}\right)}{\left[E_{m n}\left(s^{\prime}\right)\right]^{2}}\right] \mathrm{d} s^{\prime}\right\} \cdot(33)
\end{aligned}
$$

Para entendermos o comportamento da dinâmica dos coeficientes no regime adiabático, analisaremos a equação 33 (especificamente os termos que tem dependência explícita de $\tau_{\max }$ ) para tempos muito longos. Na integral no lado direito, o termo $\partial_{s^{\prime}}\left\{\frac{F_{m n}\left(s^{\prime}\right)}{\left[E_{m n}\left(s^{\prime}\right)\right]^{2}}\right\}$ é integrável no intervalo de $[0, s]$, e como uma consequência do Lema de Riemann-Lebesgue [34, 35, teremos

$$
\lim _{\tau_{m a x} \rightarrow \infty} \int_{0}^{s} e^{i \tau_{\max } \omega_{m n}\left(s^{\prime}\right)} \partial_{s^{\prime}}\left\{\frac{F_{m n}\left(s^{\prime}\right)}{\left[E_{m n}\left(s^{\prime}\right)\right]^{2}}\right\} \mathrm{d} s^{\prime}=0 .
$$


No termo entre chaves, para $\tau_{\max }$ muito longo, esperamos que ele se torne cada vez mais negligenciável, ou seja

$$
\max _{0<s<1}\left|\frac{1}{\tau_{\max }} \frac{F_{m n}\left(s^{\prime}\right)}{\left[E_{m n}\left(s^{\prime}\right)\right]^{2}}\right| \ll 1,
$$

e assim derivamos a condição de adiabaticidade

$$
\tau_{\max } \gg \max _{0<s<1}\left|\frac{\left\langle\psi_{m}(s)\left|\partial_{s} \hat{H}\right| \psi_{m}(s)\right\rangle}{\left[E_{n}(s)-E_{m}(s)\right]^{2}}\right|,
$$

ou então, como também aparece na literatura,

$$
\max _{0<s<1}\left|\frac{1}{\tau_{\max }} \frac{F_{m n}\left(s^{\prime}\right)}{\left[E_{m n}\left(s^{\prime}\right)\right]^{2}}\right| \leq \epsilon,
$$

sendo $\epsilon \ll 1$, uma quantidade arbitrariamente pequena.

Podemos extrair da equação $(36)$ que o tempo total da evolução adiabática é inversamente proporcional ao quadrado da diferença de energia ao longo do tempo entre os autoestados considerados. Assim, se satisfizermos a condição dada pela equação (36), a equação 33 se reduzirá à equação 18 .

A partir da condição de adiabaticidade dada pela equação (37), podemos escrever uma condição de adiabaticidade local. Para isso precisamos definir primeiramente a velocidade adiabática.

Definição (Velocidade Adiabática). Seja s uma curva parametrizada pela variável tempo, $t$, tal que

$$
s:\left\{t \in\left[0, \tau_{\max }\right] \longmapsto[0,1] \in \mathbb{R}, s=s(t)\right\},
$$

com $s(0)=0, s\left(\tau_{\max }\right)=1$. A velocidade adiabática é definida como

$$
v(t) \equiv \frac{\mathrm{d} s}{\mathrm{~d} t},
$$

de forma que essa grandeza representa a velocidade em que se varia a curva $s(t)$ em função do tempo $t$.

Assim, podemos dizer que, usando a equação (37), a condição adiabática local (em cada instante de tempo) deve satisfazer

$$
\frac{1}{v(s)}=\frac{\mathrm{d} t}{\mathrm{~d} s} \leq \frac{F_{m n}(s)}{\epsilon E_{m n}(s)^{2}}, \quad \forall s \in[0,1] .
$$

Nessa abordagem pela qual derivamos a condição adiabática tradicional, o teorema adiabático só garante que o sistema esteja com certeza (probabilidade igual a 1) no estado fundamental instantâneo durante toda a evolução quando o tempo total da evolução, $\tau_{\max }$, tender ao infinito. Com isso, a condição dada pela equação 36 não é necessária ou suficiente para que ocorra adiabaticidade. Em outras palavras, a Fidelidade,

$$
\mathcal{F}=\left|\left\langle\psi\left(\tau_{\max }\right) \mid \Psi\left(\tau_{\max }\right)\right\rangle\right|,
$$

entre o estado $\left|\psi\left(\tau_{\max }\right)\right\rangle$ evoluído até o tempo $\tau_{\max } \mathrm{e}$ o estado desejado $\left|\Psi\left(\tau_{\max }\right)\right\rangle$, que é o autoestado fundamental do Hamiltoniano final, somente será máxima
$(\mathcal{F}=1)$ tomando-se o limite do tempo de interpolação tendendo ao infinito. Além disso, há condições de adiabaticidade mais rigorosas [36, 37] que não serão descritas aqui pois fogem do nosso objetivo.

A aproximação adiabática tradicional, já demonstrada, é satisfatória para evidenciar a forte relação entre o tempo de evolução e a diferença de energia entre os autoestados de um sistema. Ela nos dá o direcionamento de como proceder com a execução de um algoritmo. Como exemplo, a empresa D-Wave usa do paradigma adiabático com tempo de evolução da ordem de dezenas de microssegundos 38 .

É relevante dizermos que além de fornecer os fundamentos para a CQA, o teorema adiabático é utilizado em diversos sistemas quânticos, como em cruzamento de níveis de energia [39], teoria quântica de campos [40, termodinâmica quântica 41] e técnicas como STIRAP ${ }^{3}$ pois essa classe de processos preserva informação para que seja possível saber previamente em qual autoestado o sistema se encontra [42. Ele é usando inclusive em computação quântica por portas lógicas, pois as operações lógicas devem ser feitas de modo que o sistema não seja perturbado.

\section{Aplicações da Computação Adiabática}

Como uma aplicação prática na computação quântica adiabática, iremos analisar o algoritmo de Grover, que apesar de simples é muito poderoso possuindo um ganho quadrático de eficiência em relação ao seu análogo clássico, e também o problema do caixeiro viajante sendo aplicado no computador da D-Wave.

\subsection{Algoritmo de Grover Adiabático}

Para buscar um item em um banco de dados desordenado, os algoritmos clássicos possuem uma ordem de complexidade proporcional ao número de elementos, $N$, contidos em um banco de dados, ou seja, $O(N)$ passos.

No entanto, a computação quântica nos fornece um algoritmo, chamado algoritmo de Grover [26], que possui ordem de complexidade igual $O(\sqrt{N})$, isto é, ganho quadrático em relação ao seu análogo clássico, sendo um marco na história da computação quântica. Este algoritmo, como indicado na Figura 2(a), parte de um sistema cujo estado está em uma superposição simétrica de todos os elementos possíveis do banco de dados. $\mathrm{O}$ Algoritmo de Grover é dividido em duas operações, como mostrado passo a passo na Figura 2 A primeira operação consiste na aplicação do operador Oráculo, que introduz uma fase relativa $(-1)$ na amplitude de probabilidade do estado que codifica o objeto a ser buscado, como representado na Figura 2(c). A segunda operação é a inversão em torno da média, onde a amplitude de probabilidade em cada estado aumenta (diminui) quando

\footnotetext{
3 Stimulated Raman adiabatic passage (Passagem adiabática estimulada por Raman, em traduação livre).
} 


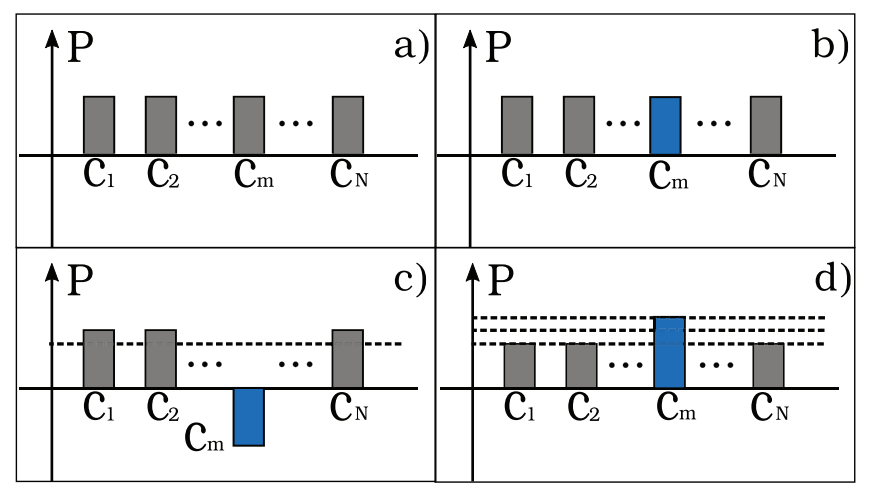

Figura 2: Representação esquemática de apenas uma aplicação do operador de Grover em um estado simétrico. No eixo vertical temos a amplitude de probabilidade dos estados, sendo cada barra (no eixo horizontal) correspondente ao $i$-ésimo estado, com $i=1, \ldots, N$. A Figura a) representa a situação inicial, em que todos os estados da base possuem a mesma amplitude de probabilidade. Na Figura $b$ ) o item que buscamos aparece marcado em azul, indicado por $\mathrm{C}_{m}$. A Figura c) indica a operação de inversão do sinal da amplitude de probabilidade do estado marcado e também, através da linha pontilhada, a média das amplitudes de probabilidades de todos os estados. Por fim, a Figura d) mostra a operação de inversão em torno da média e o incremento e decremento dos valores das amplitudes de probabilidades dos estados (linhas pontilhadas).

a amplitude antes da operação estiver abaixo (acima) da amplitude média de probabilidade. Assim, repetimos essas duas operações por cerca de $\sqrt{N}$ vezes, com $N$ sendo número de total de estados (itens no banco de dados), e em sequência medimos a probabilidade de cada estado do sistema, o estado buscado deve aparecer com probabilidade muito próxima de 1 . Outras referências sobre esse algoritmo de computação quântica de portas lógicas podem ser encontradas nas referências [9, 43.

Como o teorema da equivalência entre os dois paradigmas de computação quântica prevê que os dois são análogos [30, 44], foi encontrado uma versão adiabática para o algoritmo de busca, que chamaremos de algoritmo de Grover adiabático [4, 46. Nele, a análise de complexidade, se dá no tempo de evolução do Hamiltoniano, que é proporcional à raiz quadrada do número de itens no banco de dados, assim $\tau_{\max } \propto \sqrt{N}$.

A descrição do algoritmo de Grover adiabático começa com a definição do Hamiltoniano inicial 47,

$$
\hat{H}_{0}=\mathrm{I}-|\phi\rangle\langle\phi|,
$$

onde

$$
|\phi\rangle=\frac{1}{\sqrt{N}} \sum_{i=0}^{N-1}|i\rangle,
$$

sendo I a matriz identidade e $|i\rangle$ o $i$-ésimo autoestado da base computacional. Esse Hamiltoniano inicial coloca todos os itens do banco de dados em uma superposição simétrica, ou seja, no tempo $t=0$ todos os autoestados de $\hat{H}$ possuem a mesma probabilidade de serem medidos, por consequência a mesma energia. Sobre o seu espectro, o Hamiltoniano inicial possui energia do estado fundamental igual a 0 e os outros $N-1$ estados degenerados possuem autovalores iguais a 1 .

Consideremos então que o objeto que queremos encontrar em nossa busca é codificado pelo autoestado $|m\rangle$, que deverá ser o estado marcado pelo Hamiltoniano final, para isso, o definimos como

$$
\hat{H}_{f}=\mathrm{I}-|m\rangle\langle m|,
$$

que também tem estado fundamental, autoestado $|m\rangle$, com energia igual a 0 e os outros $N-1$ autoestados degenerados com energia igual a 1 . Com isso podemos escrever nosso Hamiltoniano dependente do tempo

$$
\hat{H}(t)=A(s)(\mathrm{I}-|\phi\rangle\langle\phi|)+B(s)(\mathrm{I}-|m\rangle\langle m|),
$$

onde $A(s)$ e $B(s)$ são funções dependentes de $s$, características de cada problema, mas com as condições de contorno dadas pela equação 2. Para o problema de busca de Grover usaremos, em um primeiro momento, a parametrização linear dada por $A(s)=1-s$ e $B(s)=s$. Reparemos que a equação 45 tem a forma da equação geral para computação adiabática, equação (3). Agora, escrito dessa forma podemos encontrar o espectro de $\hat{H}(t)$, equação 45 , de forma analítica.

Podemos definir um subespaço do Hamiltoniano dado por $\left\{|m\rangle,\left|m^{\perp}\right\rangle\right\}$. Nesse subespaço, os elementos de matriz do Hamiltoniano são dados por

$$
\begin{aligned}
\langle m|\hat{H}| m\rangle & =-(1-s)\left(1-\frac{1}{N}\right), \\
\left\langle m|\hat{H}| m^{\perp}\right\rangle & =-(1-s) \frac{1}{\sqrt{N}} \sqrt{1-\frac{1}{N}}, \\
\left\langle m^{\perp}|\hat{H}| m^{\perp}\right\rangle & =1-(1-s)\left(1-\frac{1}{N}\right) .
\end{aligned}
$$

A diagonalização do Hamiltoniano no subespaço $\left\{|m\rangle,\left|m^{\perp}\right\rangle\right\}$ resulta nos autovalores

$$
\lambda_{0,1}=\frac{1 \pm E_{01}}{2}
$$

onde

$$
E_{01}=\sqrt{(1+2 s)^{2}+\frac{4}{N} s(1-s)}
$$

é o gap de energia entre o estado fundamental e o primeiro estado excitado. A Figura 3 mostra os autovalores instantâneos do Hamiltoniano em função de $s\left(t / \tau_{\max }\right)$ onde o gap mínimo ocorre em $s=1 / 2$, sendo $E_{01}(1 / 2)=1 / \sqrt{N}$. Para esse sistema, podemos mostrar, através da equação 46, que

$$
\left|\left\langle\psi_{m}\left|\partial_{s} \hat{H}\right| \psi_{n}\right\rangle\right| \leq 1
$$




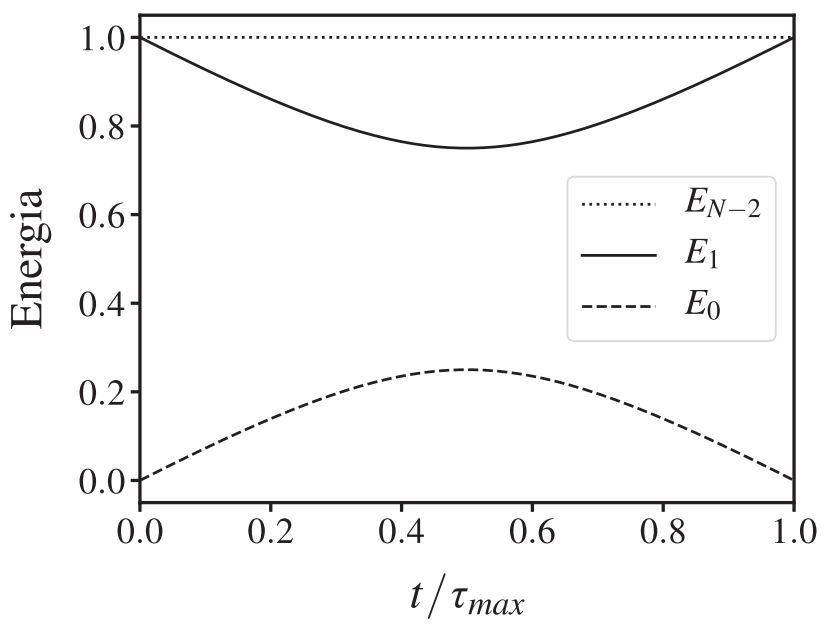

Figura 3: Autoenergias instantâneas em função do tempo parametrizado $s=t / \tau_{\max }$ associadas ao algoritmo de Grover para o caso com 4 q-bits, ou seja 16 itens no banco de dados, e interpolação $A(s)=1-s$ e $B(s)=s . E_{0}$ representa o estado fundamental (linha tracejada), $E_{1}$ é o primeiro estado excitado (linha contínua) e $E_{N-2}$ é a energia dos estados degenerados (linha pontilhada).

de forma que, para esse sistema, o tempo total de integração é regido pelo gap. Assim, o tempo total de integração é calculado usando a condição adiabática, equação (36),

$$
\begin{array}{r}
\tau_{\max } \geq\left.\frac{1}{\epsilon} \frac{1}{E_{01}(s)^{2}}\right|_{s=1 / 2}, \\
\therefore \tau_{\max } \geq \frac{N}{\epsilon}
\end{array}
$$

com $\epsilon \ll 1$, como definido na equação (37). Uma dúvida que poderia surgir aqui é em relação a constante $\epsilon$ pois, como a definimos arbitrariamente pequena, então, em tese, poderíamos controlá-la para obter qualquer tempo de integração. Todavia, tratando esse problema com mais cautela, como feito em [16], vemos que essa constante também escala, de forma que o tempo assintótico de integração continua sendo da ordem de $N$, isso é, continua possuindo ordem de complexidade igual à $\mathcal{O}(N)$. Essa discussão nos permite concluir que o algoritmo de Grover, quando formulada dessa forma, não traz ganho em relação ao seu análogo clássico.

Não obstante, é possível encontrar uma maneira de otimizar a interpolação adiabática. Podemos definir, a partir da condição adiabática local, equação 40, uma equação diferencial,

$$
\frac{\mathrm{d} t}{\mathrm{~d} s}=\frac{\left\langle\psi_{0}\left|\partial_{s} \hat{H}\right| \psi_{1}\right\rangle}{\epsilon E_{01}^{2}},
$$

Assim, usando as equações 48 e 49, escrevemos

$$
\mathrm{d} t=\frac{\mathrm{d} s}{\epsilon E_{01}^{2}}=\frac{\mathrm{d} s}{\epsilon\left[(1+2 s)^{2}+\frac{4}{N} s(1-s)\right]},
$$

que integrada de 0 a $s$ resulta em

$$
\begin{aligned}
t= & \frac{1}{2 \epsilon} \frac{N}{\sqrt{N-1}}\{\arctan [\sqrt{N-1}(2 s-1)] \\
& +\arctan (\sqrt{N-1})\} .
\end{aligned}
$$

Realizando sua expansão em série de potências para $N \gg 1$ e calculando-a no tempo $s=1$, obtemos que

$$
\tau_{\max }=t(s=1)=\frac{\pi}{2 \epsilon} \sqrt{N},
$$

de forma que encontramos um algoritmo de busca adiabático que possui o mesmo desempenho do algoritmo por portas, como esperado. Este ganho em relação a parametrização é justificado pelo fato que essa curva otimizada acelera (desacelera) a dinâmica do sistema quando o gap diminui (aumenta). Esse resultado indica que a eficiência da computação adiabática depende intrinsecamente da parametrização da interpolação escolhida.

A Figura 4 mostra a fidelidade, definida pela equação 41, em função do tempo máximo de integração, em unidades arbitrárias, para as duas parametrizações do algoritmo de Grover para o caso com 4 q-bits, ou seja $N=16$ itens no banco de dados. Essa Figura nos permite uma verificação numérica do ganho temporal quando se usa uma função de interpolação mais adequada.

Em adição, na literatura podemos encontrar um método, baseado em cálculo variacional, que visa formalizar a busca pelas funções que reproduzem a interpolação adiabática mais rápido. Em outras palavras,

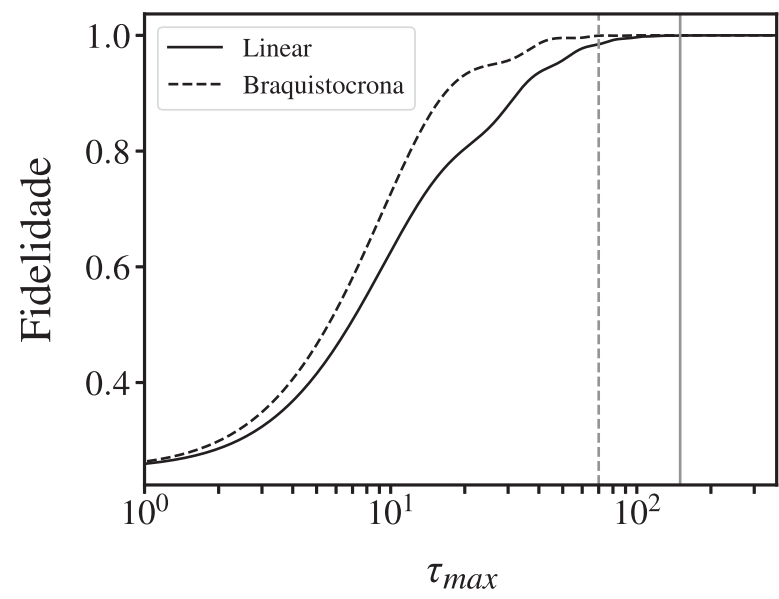

Figura 4: Fidelidade do estado do sistema, obtido após uma evolução completa até $t=\tau_{\max }$, com relação ao estado fundamental do Hamiltoniano $\hat{H}_{f}$ em função do tempo de annealing $\left(\tau_{\max }\right)$, associada ao algoritmo de Grover para o caso com 4 q-bits, ou seja 16 itens no banco de dados. Temos duas interpolações: a linha contínua refere-se à interpolação linear, cuja fidelidade igual a 0.995 é atingida para $\tau_{\max }=150$, e a linha tracejada refere-se à interpolação advinda do cálculo variacional, que permite atingir a mesma fidelidade com tempo total $\tau_{\max }=70$. Notemos que o tempo está em unidades arbitrárias. 
um método que encontra a braquistócrona do processo adiabático [48]. O entendimento da braquistócrona continua sendo um objeto de estudo, pois para cada sistema quântico temos uma parametrização ótima diferente, além disso, esse método para encontrar a melhor parametrização pode ser estendido para sistemas quânticos abertos [49].

\subsection{Problema do caixeiro viajante}

Suponha que um caixeiro (ou carteiro) precise realizar diversas entregas, cada uma em uma cidade diferente. O problema do caixeiro viajante trata-se de uma tarefa para encontrar a menor rota que passe por um conjunto de cidades diferentes apenas uma única vez antes que o caixeiro retorne à cidade de início. Esse problema é do tipo NP-difícil, o que significa que o seu espaço de solução cresce de forma fatorial com o número de cidades $N$ a serem visitadas 9. Os algoritmos exatos existentes criam estratégias para buscar soluções que resolvam o problema do caixeiro viajante, alcançando a solução ótima com uma ordem de complexidade igual à $\mathrm{O}\left(N^{2} 2^{N}\right)$, o que é muito custoso computacionalmente [50, 51].

Podemos transformar tal problema possibilitando a resolução em um computador quântico da D-Wave [52]. Para fazê-lo, definimos a variável binária $x_{i, l}$ que assume o valor 1 quando o caixeiro está na cidade $i$ no instante de tempo $l$, e o valor 0 caso contrário. Com isso podemos definir o Hamiltoniano de spins

$$
H_{\text {Ising }}=\sum_{i, j \in E} J_{i j} \sum_{l}^{N} x_{i, l} x_{j, l+1},
$$

com $J_{i j}$ sendo a distância entre as cidades vizinhas $i$ e $j$ e $E$ o conjunto de conexões entre as cidade.

Como temos $N$ intervalos de tempo para $N$ cidades, serão necessários $N^{2}$ q-bits para uma lista com $N$ cidades. Isto dificulta a implementação dessa abordagem em computadores clássicos, já que o espaço de Hilbert dessa classe de problemas, trabalhando com q-bits, cresce da forma $2^{N^{2}}$, de forma que, em computadores pessoais, essa implementação fica limitada em até 4 cidades.

Há outras questões que devemos levar em conta como a inclusão de condições de contorno que possibilitem o computador a saber se o caixeiro passou duas vezes pela mesma cidade, ou se ele está parado em uma cidade por mais de um intervalo de tempo. Como o annealing quântico leva o sistema para o estado de menor energia, podemos acrescentar penalidade energéticas para os casos citados acima como:

$$
\begin{aligned}
\hat{H}_{\text {penal }}= & \lambda\left[\sum_{l=1}^{N}\left(1-\sum_{i=1}^{N} x_{i, l}\right)^{2}+\sum_{i=1}^{N}\left(1-\sum_{l=1}^{N} x_{i, l}\right)^{2}\right. \\
& \left.+\sum_{(i, j) \notin E} \sum_{l=1}^{N} x_{i, l} x_{j, l+1}\right]
\end{aligned}
$$

onde o primeiro termo penaliza a passagem pela mesma cidade em tempos diferentes, o segundo penaliza estados com duas cidades em um mesmo tempo e o terceiro penaliza a passagem por conexões que não existem. A constante $\lambda$ irá ditar a intensidade de $\hat{H}_{\text {penal }}$. Notemos que o Hamiltoniano $\hat{H}_{\text {penal }}$ ainda se encaixa num problema QUBO e possui seu mínimo em zero apenas quando as condições do problema do caixeiro viajante são satisfeitas.

A teoria sobre interpolações adiabática que aqui desenvolvemos é específica para sistemas quânticos, de forma que para transformar a representação do caixeiro viajante dada pela Hamiltoniana clássica em uma representação dada por um Hamiltoniano quântico, basta fazer a troca da variável $x_{i, j}$ para $\left(1+\sigma_{i, j}^{z}\right) / 2$, com $\sigma_{i, j}^{z}$ sendo a matriz de Pauli-Z (com autovalores 1 e -1 ) que representa a cidade $i$ no tempo $j$. Fazendo isso introduzimos os operadores de spin, atributos quânticos, e ainda mantemos a propriedade binária das variáveis. Essa troca gera um Hamiltoniano com autoestados da forma, $\left|x_{0,0}, x_{1,0}, x_{2,0}, \ldots, x_{N, N}\right\rangle$, com $x_{i, j}$ sendo a variável binária definida anteriormente. Assim, o Hamiltoniano final fica escrito como

$$
\hat{H}(s)=A(s) \sum_{i} \hat{\sigma}_{i}^{x}+B(s)\left(\hat{H}_{\text {ising }}+\hat{H}_{\text {penal }}\right),
$$

onde, como na equação (3), o primeiro termo é o Hamiltoniano inicial que é obtido aplicando-se um campo magnético alinhado na direção $x$, e a interpolação é feita desligando-o lentamente enquanto aumenta-se a intensidade das conexões entre os spins, que é descrito pelo Hamiltoniano final, dado pelo segundo termo.

Para um problema com seis cidades colocadas randomicamente no espaço conseguimos encontrar a solução exata usando o computador da D-Wave, como está mostrado no repositório público [53]. O problema que resolvemos está mostrado na Figura 5. Para realizar tal tarefa foi necessário executar o algoritmo 500 vezes, sendo que cada execução levou 20 microssegundos. Um outro parâmetro ajustável é um fator multiplicativo do Hamiltoniano penal, indicador por $\lambda$ na equação (56).
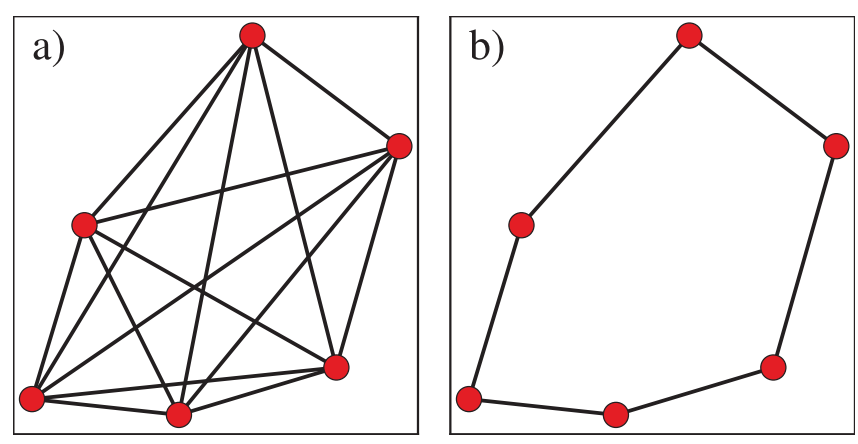

Figura 5: Representação de 6 cidades distribuídas aleatoriamente pelo espaço. Figura a) mostra o problema com todas as suas possíveis conexões e a Figura b) mostra a solução obtida pelo computador quântico da D-Wave. 
A empresa pré-define o valor de $\lambda=\lambda_{0}$, sendo $\lambda_{0}$ calculado pela expressão

$$
\lambda_{0}=\frac{N \times \sum J_{i j}}{N_{c}}
$$

que é a soma dos acoplamentos, $J_{i j}$, vezes o número de cidades, dividido pelo número de conexões $\left(N_{c}\right)$ entre os cidades. Entretanto, o parâmetro $\lambda$ pode ser alterado pelo usuário e a empresa indica que o algoritmo produz bons resultados quando ele assume valores entre entre $75 \%$ e $150 \%$ do valor indicado pela equação (58). Em nosso problema, o sistema que encontrou a solução possuía $\lambda=1.05 \lambda_{0}$.

Dado que esse problema requer muitos q-bits, isso é, $N^{2}$ q-bits para $N$ cidades mais os q-bits necessários para o processo de minor embedding, é possível abordar apenas até 9 cidades no D-Wave 2000Q [52. No entanto, podemos criar uma estratégia heurística para resolver problemas com mais cidade dividindo e conquistando. Essa estratégia consiste em dividirmos um problema de caixeiro viajante em subproblemas e depois conectar esses subproblemas como se fossem um núcleos e tratar esses núcleos como um outro caixeiro viajante. As referências [52 54] mostram o uso do computador quântico para resolver problemas deste tipo.

\section{D-Wave: Um Panorama da Tecnologia}

Nessa Seção descrevemos as características do annealer quântico da D-Wave mostrando alguns aspectos de sua engenharia, limitações e vantagens [55].

As unidades de processamento quântico (chips) dos computadores da D-Wave são compostos por q-bits, que são construídos usando-se CCJJ rf-SQUIDs (do inglês compound-compound Josephson-junction radio frequency superconducting quantum interferences devices) que são circuitos supercondutores de dimensões microscópicas que usam de junções Josephson e operam em radiofrequência [56 58].

Em materiais condutores normais, elétrons repelem-se devido a suas cargas negativas. Porém, quando tratamos de elétrons em materiais supercondutores, existe uma interação entre elétrons e fônons decorrente dos modos de vibração da rede cristalina do material que cria uma interação atrativa entre os elétrons. Assim elétrons de spins opostos se emparelham, os chamados "pares de Cooper", que possuem uma função de onda simétrica pois se comportam como bósons, acarretando em uma falta de resistência ao movimento coletivo, criando assim o fenômeno da supercondutividade [59]. Portanto, os portadores de carga em um supercondutor são pares de Cooper, que dão origem a correntes não dissipativas em materiais supercondutores altamente sensíveis a campos magnéticos externos, permitindo assim o desenvolvimento de dispositivos como os SQUIDs. Um SQUID é composto por um loop supercondutor interrompido por junções Josepheson (JJ), que são constituídas de isolantes finos (barreira de potencial). No supercondutor as cargas negativas se movem livremente. Porém, quando elas colidem com uma JJ há uma possibilidade de tunelamento, que, por sua vez, altera a fase da função de onda do par eletrônico dentro do supercondutor. Desse modo, há uma diferença de fase entre a onda refletida e a transmitida pela JJ, a qual afeta diretamente o sentido da corrente no loop supercondutor. Dessa forma, é possível controlar (externamente) a voltagem dentro do dispositivo para reger o tunelamento desses pares eletrônicos e assim mudar o sentido da corrente que passa pela JJ, criando uma superposição quântica das correntes.

Os q-bits da D-Wave tem suas conexões mediadas por outros dispositivos supercondutores, os CJJ rf SQUIDs (compound Josephson-junction radio frequency superconducting quantum interferences devices), ou seja, o acoplamento entre os q-bits é feito usando um dispositivo supercondutor muito similar àquele dos q-bits [57, 60]. Devido a limitações físicas, isso é, pelas restrições impostas pelo tamanho e formato dos q-bits, a forma com que os q-bits se conectam na rede é definida por diferentes topologias. A Figura 6(a) mostra a topologia do computador 2000Q lançado em 2017 que possui 2048 q-bits interligados 6 a 6 pela topologia batizada como Chimera, já a Figura 6(b) mostra a topologia do computador lançado em 2020, o Advantage, que possui mais de 5000 q-bits interligados 15 a 15 pela topologia Pegasus. Da comparação entre as topologias é notório que a última, além de possuir uma quantidade maior de q-bits, apresenta uma conectividade muito maior entre eles. $\mathrm{Na}$ arquitetura do computador quântico da D-Wave o Hamiltoniano implementado não consegue codificar todos os problemas que envolvem portas lógicas, mas apenas problemas de otimização de corte máximo [62, que são problemas de otimização combinatória binária. Esse tipo de problema inclui uma grande diversidade de tarefas reais e de interesse industrial, como, por exemplo, o problema do caixeiro viajante [63], o problema de inspeção de rotas 64 e resolução de sistemas de equações lineares.

O chip processador da D-Wave, como dito anteriormente, possui uma topologia que conecta os q-bits disponíveis o que gera um certo problema, pois, em geral, o problema a ser resolvido não possui essas mesmas conexões. Para contornar essa adversidade é necessário uma etapa intermediária antes de realizar o annealing, que é conhecida como minor embedding [65, que pode ser realizada de forma automática dos computadores da D-Wave ou manualmente pelo usuário [66]. Essa estratégia mapeia a topologia do problema de otimização a ser resolvido na topologia do computador quântico e com isso vem um custo e algumas implicações. Em primeiro lugar esse problema, que é da classe $N P$ [67, é executado em um computador clássico usando algoritmos heurísticos 68 70, depois temos que a qualidade 


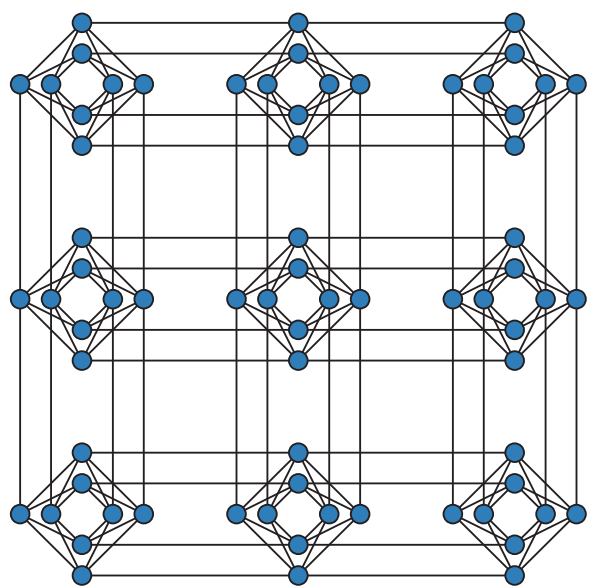

(a) Topologia Chimera.

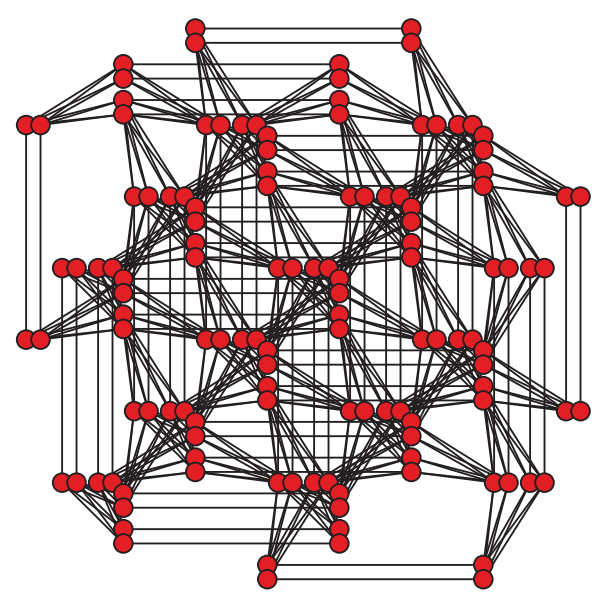

(b) Topologia Pegasus.

Figura 6: Comparação entre as topologias das duas gerações mais novas de annealing para um subgrupo com 96 q-bits. (a) Topologia Chimera do computador 2000Q lançado em 2017. (b) Topologia Pegasus do computador Advantage lançado em 2020. Fizemos essa figura usando a biblioteca open source [61.

da solução obtida depende da qualidade do embedding, ou seja, embeddings diferentes podem gerar soluções diferentes. Por fim, a realização desse mapeamento aumenta o número de q-bits requeridos pelos sistema, em alguns casos, quadraticamente, o que diminui a capacidade de resolver problemas maiores, portanto mais reais.

Apesar da tecnologia dos computadores quânticos da D-Wave ainda não permitir resultados melhores que os dos computadores clássicos, tal tecnologia já consegue reproduzir resultados qualitativamente similares [71, ou até com algum ganho significativo, como foi reportado em 72 em que um problema específico de uma empresa canadense, resolvido em 25 horas por um computador clássico, pôde ser resolvido em 2 horas pelo D-Wave Advantage. Entretanto, diversos estudos demonstram que os computadores quânticos com técnica de annealing são capazes de resolver os problemas propostos e que nas próximas gerações de computadores será possível aumentar o tamanho das entradas dos problemas, como consequência será obtido uma superioridade nítida em relação aos computadores clássicos [52, 71, 73, 74].

Além disso, podemos apontar outras vantagens inerentes à computação quântica adiabática, como a geração de números genuinamente aleatórios 75 e a economia de energia, pois apesar da necessidade de resfriamento a temperaturas próximas de zero absoluto, o gasto de energia não aumenta com a evolução da tecnologia, assim sendo mais econômica que os supercomputadores clássicos [76].

Existem duas possibilidades de acesso do público em geral aos computadores quânticos da D-Wave, no entanto nenhuma é gratuita. É possível usar seus computadores quânticos por meio da plataforma Amazon Braket [77] ou por meio da plataforma Leap, da própria D-Wave 78 .

\section{Conclusão}

Este artigo teve como propósito embasar o funcionamento da teoria da computação quântica adiabática e possibilitar o entendimento dos recentes avanços nesta área. Expusemos o funcionamento da tecnologia usada pela empresa de computação quântica D-Wave, baseada na teoria do annealing quântico que, diferentemente do caso clássico, não envolve variações adiabáticas da temperatura, mas sim uma evolução temporal adiabática de um determinado Hamiltoniano/sistema. Mostramos o paradigma da computação adiabática provando o teorema adiabático e derivando uma condição de adiabaticidade tradicional. Como uma primeira aplicação foi descrito o algoritmo de Grover adiabático e foi discutindo brevemente como a forma da interpolação adiabática interfere na complexidade do algoritmo. Mostramos também como é feito o processo de construção dos algoritmos, dando o exemplo do problema do caixeiro viajante.

A lei de Moore apresenta uma tendência do desenvolvimento de relacionado à industria de microchips e processamento de computadores 79. Ela mostra a projeção esperada para o número de transistores dos chips, ou seja, capacidade de processamento, no decorrer dos anos. Nos cerca de 40 anos da primeira proposta com computação quântica feita por P. Benioff e R. Feynman, vemos que o desenvolvimento nesta área cresce de forma análoga àquela mostrada pela lei de Moore, indicando que em breve poderemos falar em supremacia quântica como definida por J. Preskill [21].

\section{Agradecimentos}

Este trabalho teve o apoio do Conselho Nacional de Desenvolvimento Científico e Tecnológico (CNPq), da Coordenação de Aperfeiçoamento de Pessoal de Nível Superior (CAPES) - Código 001, e do Instituto Nacional de Ciência e Tecnologia para Informação Quântica 
(INCT-IQ/CNPq), Processo No. 465469/2014-0. C.J. V.-B. também agradece o apoio da Fundação de Pesquisa do Estado de São Paulo (FAPESP), Processo No. 2019/11999-5, e do CNPq, Processo No. 307077/2018-7.

\section{Referências}

[1] M. Davis, The undecidable: Basic papers on undecidable propositions, unsolvable problems and computable functions (Dover Publications, Mineola, 2004).

[2] J.J. Bartik, Pioneer programmer: Jean Jennings Bartik and the computer that changed the world (Truman State University Press, Kirksville, 2013).

[3] J. Backus, ACM Sigplan Notices 13, 165 (1978).

[4] P. Norton, Inside the IBM PC: access to advanced features and programming; new and enlarged (R.J. Brady, Bowie, 1986).

[5] P. Benioff, Journal of Statistical Physics 22, 563 (1980).

[6] R.P. Feynman, Int. J. Theor. Phys 21 (1982).

[7] C. Monroe, D. Meekhof, B. King, W.M. Itano e D.J. Wineland, Physical review letters 75, 4714 (1995).

[8] H. Johnston, Primeiro Computador Quântico comercial D-Wave 1, disponível em: https://web.archive.org/we b/20110515083848/http://physicsworld.com/cws/artic le/news/45960, acessado em 20/02/2021.

[9] M.A. Nielsen e I. Chuang, Quantum computation and quantum information (Cambridge University Press, Cambridge, 2011).

[10] D.P. DiVincenzo, Fortschritte der Physik: Progress of Physics 48, 771 (2000).

[11] A. Shnirman, G. Schön e Z. Hermon, Physical Review Letters 79, 2371 (1997).

[12] D. Averin, Solid State Communications 105, 659 (1998).

[13] J.I. Cirac e P. Zoller, Nature 404, 579 (2000).

[14] D. Loss e D.P. DiVincenzo, Physical Review A 57, 120 (1998).

[15] M.A. Nielsen e I.L. Chuang, em: Quantum Computation and Quantum Information, (Cambridge University Press, Cambridge, 2011).

[16] T. Albash e D.A. Lidar, Reviews of Modern Physics 90, 015002 (2018).

[17] Show your work: D-wave opens the door to performance comparisons between quantum computing architectures, disponível em: https://www.dwavesys.com/press-relea ses/show-your-work-d-wave-opens-door-performance-c omparisons-between-quantum-computing, acessado em 25/01/2021.

[18] J. Preskill, Quantum 2, 79 (2018).

[19] F. Arute, K. Arya, R. Babbush, D. Bacon, J.C. Bardin, R. Barends, R. Biswas, S. Boixo, F.G. Brandao, D.A. Buell et al., Nature 574, 505 (2019).

[20] H.S. Zhong, H. Wang, Y.H. Deng, M.C. Chen, L.C. Peng, Y.H. Luo, J. Qin, D. Wu, X. Ding, Y. Hu et al., Science 370, 1460 (2020).

[21] J. Preskill, arXiv:1203.5813 (2012).

[22] D-Wave Advantage, disponível em: https://www.dwav esys.com/d-wave-two\% E2\%84\% A2-system acessado em $18 / 01 / 2021$.
[23] L.E. Ballentine, Quantum mechanics: a modern development (World Scientific Publishing Company, Singapore, 2014).

[24] M.S. Sarandy, L.A. Wu e D.A. Lidar, Quantum Information Processing 3, 331 (2004).

[25] C.C. Chang, A. Gambhir, T.S. Humble e S. Sota, Scientific reports 9, 1 (2019).

[26] L.K. Grover, Physical review letters 79, 325 (1997).

[27] J. Roland e N.J. Cerf, Physical Review A 65, 042308 (2002).

[28] P.J. Van Laarhoven e E.H. Aarts, em: Simulated annealing: Theory and applications (Springer, Dordrecht, 1987).

[29] S. Kirkpatrick, C.D. Gelatt e M.P. Vecchi, Science 220, 671 (1983).

[30] D. Aharonov, W. Van Dam, J. Kempe, Z. Landau, S. Lloyd e O. Regev, SIAM review 50, 755 (2008).

[31] D-Wave: What is Quantum Annealing?, disponível em: https://docs.dwavesys.com/docs/latest/c_gs_2.html, acessado em 18/02/2021.

[32] M. Born e V. Fock, Zeitschrift für Physik 51, 165 (1928).

[33] T. Kato, Journal of the Physical Society of Japan 5, 435 (1950).

[34] I.S. Gradshteyn e I.M. Ryzhik, Table of integrals, series, and products (Academic Press, Cambridge, 2014).

[35] C.S. Kahane, Czechoslovak Mathematical Journal 30, 108 (1980).

[36] D. Comparat, Physical Review A 80, 012106 (2009).

[37] Z.Y. Wang e M.B. Plenio, Physical Review A 93, 052107 (2016).

[38] Anneal Schedule Variations, disponível em: https://do cs.dwavesys.com/docs/latest/c_fd_as.html, acessado em 05/10/2020.

[39] C. Zener, Proceedings of the Royal Society of London. Series A, Containing Papers of a Mathematical and Physical Character 137, 696 (1932).

[40] M. Gell-Mann e F. Low, Physical Review 84, 350 (1951).

[41] O. Abah e E. Lutz, Europhysics Letters 118, 40005 (2017).

[42] N.V. Vitanov, A.A. Rangelov, B.W. Shore e K. Bergmann, Reviews of Modern Physics 89, 015006 (2017).

[43] L.K. Grover, em: Proceedings of the twenty-eighth annual ACM symposium on Theory of Computing (Association for Computing Machinery, New York, 1996).

[44] H. Yu, Y. Huang e B. Wu, Chinese Physics Letters 35 , 110303 (2018).

[45] E. Farhi, J. Goldstone, S. Gutmann e M. Sipser, arXiv:quant-ph/0001106 (2000).

[46] E. Farhi e S. Gutmann, Physical Review A 57, 2403 (1998).

[47] J. Roland e N.J. Cerf, Phys. Rev. A 65, 042308 (2002).

[48] A. Rezakhani, W.J. Kuo, A. Hamma, D. Lidar e P. Zanardi, Physical review letters 103, 080502 (2009).

[49] A.C. Santos, C.J. Villas-Boas e R. Bachelard, Physical Review A 103, 012206 (2021).

[50] M. Held e R.M. Karp, Journal of the Society for Industrial and Applied mathematics 10, 196 (1962).

[51] R. Bellman, Journal of the ACM 9, 61 (1962).

[52] R.H. Warren, SN Applied Sciences 2, 75 (2020). 
[53] Quantum Annealing, disponível em: https://github .com/PauloJPS/QuantumAnnealing, acessado em $18 / 01 / 2021$.

[54] C. Papalitsas, T. Andronikos, K. Giannakis, G. Theocharopoulou e S. Fanarioti, Algorithms 12, 224 (2019).

[55] Technical Description of the $Q P U-D$-Wave System Documentation, disponível em: https://docs.dwave sys.com/docs/latest/doc_qpu.html, acessado em $05 / 10 / 2020$.

[56] D. Drung, C. Abmann, J. Beyer, A. Kirste, M. Peters, F. Ruede e T. Schurig, IEEE Transactions on Applied Superconductivity 17, 699 (2007).

[57] R. Harris, J. Johansson, A.J. Berkley, M.W. Johnson, T. Lanting, S. Han, P. Bunyk, E. Ladizinsky, T. Oh, I. Perminov et al., Physical Review B 81, 134510 (2010).

[58] R. Jaklevic, J. Lambe, A. Silver e J. Mercereau, Physical Review Letters 12, 159 (1964).

[59] S. Fujita, K. Ito e S. Godoy, Quantum Theory of Conducting Matter: Superconductivity (Springer-Verlag, New York, 2009).

[60] R. Harris, T. Lanting, A.J. Berkley, J. Johansson, M.W. Johnson, P. Bunyk, E. Ladizinsky, N. Ladizinsky, T. Oh e S. Han, Physical Review B 80, 052506 (2009).

[61] D-Wave Systems, disponível em: https://github.com/d wavesystems, acessado em 18/01/2021.

[62] G. Ausiello, P. Crescenzi, G. Gambosi, V. Kann, A. Marchetti-Spaccamela e M. Protasi, Complexity and approximation: Combinatorial optimization problems and their approximability properties (Springer Science, Berlin, 2012).

[63] R. Matai, S.P. Singh e M.L. Mittal, em: Traveling salesman problem: an overview of applications, formulations, and solution approaches (IntechOpen, London, 2010).

[64] H.A. Eiselt, M. Gendreau e G. Laporte, Operations Research 43, 231 (1995).

[65] V. Choi, Quantum Information Processing 7, 193 (2008).

[66] Minor Embedding d-wave handbook, disponível em: http s://docs.dwavesys.com/docs/latest/c_handbook_5.html, acessado em 12/01/2021.

[67] J. Matoušek e R. Thomas, Discrete Mathematics 108, 343 (1992).

[68] J. Cai, W.G. Macready e A. Roy, arXiv:1406.2741 (2014).

[69] E.G. Rieffel, D. Venturelli, B. O'Gorman, M.B. Do, E.M. Prystay e V.N. Smelyanskiy, Quantum Information Processing 14, 1 (2015).

[70] Z. Bian, F. Chudak, R.B. Israel, B. Lackey, W.G. Macready e A. Roy, Frontiers in ICT 3, 14 (2016).

[71] M. Ohzeki, A. Miki, M.J. Miyama e M. Terabe, arXiv:1812.01532 (2018).

[72] New Generation Improvement: d-wave advantage, disponível em: https://www.dwavesys.com/press-releases/ d-wave-announces-general-availability-first-quantum-c omputer-built-business, acessado em 12/1/2021.

[73] D. Venturelli, D.J.J. Marchand e G. Rojo, arXiv:1506.08479 (2016)

[74] C.C. Chang, A. Gambhir, T.S. Humble e S. Sota, Scientific reports 9, 1 (2019).

[75] M. Herrero-Collantes e J.C. Garcia-Escartin, Reviews of Modern Physics 89, 015004 (2017).
[76] https://www.dwavesys.com/sites/default/files/14-1005 A_D_wp_Computational_Power_Consumption_and_Spe edup.pdf, acessado em 05/10/2020.

[77] Plataforma de acesso para d-wave pela amazon aws, disponível em: https://aws.amazon.com/pt/braket/ acessado em 16/02/2021.

[78] Plataforma de acesso para d-wave, disponível em: ht tps://www.dwavesys.com/take-leap, acessado em $16 / 02 / 2021$.

[79] I.S. Oliveira, T.J. Bonagamba, R.S. Sarthour, J.C. Freitas e E.R. de Azevedo, NMR Quantum Information Processing (Elsevier, Amsterdam, 2007). 